\title{
Prognostic significance of the primary tumor site and immune indexes in patients with estrogen receptor-positive, human epidermal growth factor receptor-2-negative breast cancer
}

\author{
Xinming Song ${ }^{1 \#}$, Jianli $\mathrm{Ma}^{2 \#}$, Han Zhang ${ }^{1}$, Qingyuan Zhang ${ }^{1}$ \\ ${ }^{1}$ Department of Medical Oncology, Harbin Medical University Cancer Hospital, Harbin Medical University, Harbin, China; ${ }^{2}$ Department of \\ Radiation Oncology, Harbin Medical University Cancer Hospital, Harbin Medical University, Harbin, China \\ Contributions: (I) Conception and design: Q Zhang, X Song; (II) Administrative support: Q Zhang; (III) Provision of study materials or patients: X \\ Song, J Ma; (IV) Collection and assembly of data: X Song, J Ma; (V) Data analysis and interpretation: X Song, H Zhang; (VI) Manuscript writing: All \\ authors; (VII) Final approval of manuscript: All authors. \\ \#These authors contributed equally to this work. \\ Correspondence to: Qingyuan Zhang. Department of Medical Oncology, Harbin Medical University Cancer Hospital, Harbin Medical University, \\ Harbin 150081, China. Email: 13313612989@163.com.
}

Background: The ability to predict high risk factors for recurrence after neoadjuvant chemotherapy (NAC) is controversial. The purpose of the present study was to investigate the prognostic significance of tumor location, tumor-infiltrating lymphocyte (TIL) level, and pretreatment lymphocyte-to-monocyte ratio (LMR) in determining the survival of patients with estrogen receptor (ER)-positive, human epidermal growth factor receptor-2 (HER2)-negative breast cancer after treatment with NAC.

Methods: The clinical data of 285 ER-positive, HER2-negative patients with clinical stage II-III breast cancer were analyzed from January 2009 to January 2015. To explore the prognostic factors for ERpositive, HER2-negative patients, we combined the conventional clinicopathological prognostic factors with tumor location, pretreatment LMR, and TIL. In addition, samples from 79 patients, who did not achieve pathological complete response (pCR) testing after NAC, were selected for hematoxylin-eosin (HE) staining to analyze the effect of TIL on prognosis.

Results: An LMR > 5.2 was correlated with better 5-year disease-free survival (DFS) and overall survival (OS; $\mathrm{P}<0.001$ and $\mathrm{P}<0.001$, respectively). Patients with lower-inner/central quadrant tumors had lower 5 -year DFS and OS than patients with tumors in the other quadrants $(\mathrm{P}=0.012$ and $\mathrm{P}=0.048)$. Patients with a lower TIL level $(\leq 10 \%)$ had better 5 -year DFS than patients with a higher TIL level $(\mathrm{P}=0.010)$. According to the results of the multivariate analyses, tumor location was an independent prognostic factor for 5 -year DFS ( $\mathrm{P}=0.021)$. Pretreatment LMR was associated with both 5 -year DFS and $\mathrm{OS}(\mathrm{P}<0.001$ and $\mathrm{P}<0.001$, respectively). In the subgroup analysis stratified by TIL level, the TIL level and the initial clinical stage were associated with 5-year DFS ( $\mathrm{P}=0.027$ and $\mathrm{P}<0.001$, respectively).

Conclusions: We explored the prognostic significance of the tumor site, TIL level, and pretreatment LMR level for ER-positive, HER2-negative patients. We concluded that the lower-inner/central quadrant tumors, TIL $>10 \%$, and pretreatment LMR level $\leq 5.2$ were correlated with a poor prognosis. More aggressive NAC and/or endocrine therapy with internal mammary node radiotherapy (IMN-RT) should be administered to address the relatively poor prognosis of patients with breast carcinoma presenting the aforementioned adverse factors.

Keywords: Breast cancer; tumor site; neoadjuvant chemotherapy (NAC); lymphocyte-to-monocyte ratio (LMR); tumor-infiltrating lymphocyte (TIL); human epidermal growth factor receptor-2 (HER2); estrogen receptor (ER)

Submitted Jun 29, 2020. Accepted for publication Sep 16, 2020.

doi: 10.21037 /gs-20-622

View this article at: http://dx.doi.org/10.21037/gs-20-622

(c) Gland Surgery. All rights reserved. 


\section{Introduction}

Breast cancer is the most common malignant tumor diagnosed in women worldwide. The treatment of the breast cancer had developed from the surgery only to multidisciplinary therapies. Generations of the moleculetargeted drugs have been developed. CDK4/6 inhibitor or the estrogen receptor (ER) antagonists also pave a new way for treating the HR positive ones. Although significant progress has been made in its treatment, breast cancer, still has a high tendency of relapse, with some patients ultimately progressing to late-stage breast cancer. Consequently, the identification of proper prognostic factors is highly important, and clinicians and researchers have performed numerous studies to investigate more precise prognostic factors that will guide therapy for NAC patients. The histologic grade, initial clinical stage, and different clinicopathological features have all been recognized as traditional clinicopathological factors. Unconventional prognosis values which were also under exploration by the researchers compromised many types. For example, miR222, miR-29a and the miR-34a were representative of the chemo-resistant markers. Small molecule changes (IL-6, IL8, MMP-9) may provide new breakthroughs in researching how to acquire better prognosis and survival Patients should be treated individually according to different recurrence risks. However, traditional clinicopathological features are not sufficient to predict all relapse cases due to the complexity of patients' conditions. Therefore, it is important to identify more definite prognostic factors.

Until now, many researchers tried their best to find more accurate and detailed prognosis factor for the NAC patients. There are several major categories of prognosis variables. The clinicopathological factors mainly comprised of ki-67, clinical stage, PCR and ypN0 et al. Biochemical prognostic factors and immune markers also played an important role in predicting the outcome. When it comes to the biochemical prognostic factors aspect, IL-10, CTC and miRNA had attached lots of attention. TILs and peripheral blood markers such as the LMR, NLR and PLR were Representativeness of the immune markers. Even a long course of therapy, breast cancer can recur. Currently available retrospective studies have attempted to identify new prognostic factors. The estrogen receptor (ER)-positive, human epidermal growth factor receptor-2 (HER2)-negative subgroup is a unique subtype of breast cancer, as it is recognized as a cohort of tumors with a confusing prognosis and distinct immune features. The prediction and comprehension of the value of traditional prognostic factors (such as PCR) for this subgroup of patients is challenging and had attracted lots of attention. There still exist the controversy that the endocrine therapy may be superior to chemotherapy for this subgroup patients. The non-traditional prognostic factors maybe more suitable for this subgroup. Among the non-traditional prognostic factors, we mainly focused on the primary tumor site, lymphocyte-to-monocyte ratio (LMR), and tumor-infiltrating lymphocyte (TIL) level. Researchers have extensively studied these three variables (particularly the last two) in recent years. The primary tumor site has long been recognized as a controversial variable, and most studies examining this parameter have concluded that breast cancer in the inner quadrant results in worse overall survival (OS) than tumors located in the outer quadrant (1-3). The role of the immune reaction in tumorigenesis and tumor development have been the focus of researchers. The LMR and TIL level are the most common and representative inflammatory response markers, as they indirectly indicate the status of the immune system of the patients. The LMR is defined as the number of peripheral white blood cells divided by the number of peripheral blood monocytes. In their retrospective study, Peng et al. revealed that a higher LMR is related to a higher pathological complete response (pCR) rate for the patients undergoing neoadjuvant chemotherapy (NAC) (4). Regarding the other inflammatory response marker, the TIL is mainly comprised of T cells, B cells, natural killer cells, and dendritic cells. This has also been a well-researched topic, with Loi et al. postulating that a high TIL level is an independent factor, indicating better prognosis for patients in the triple-negative and the HER2enriched subgroup (5).

To the best of our knowledge, the present retrospective study is the first to explore the prognostic significance of the primary tumor site, TIL level, and LMR for ERpositive, HER2-negative patients. Previous articles mainly paid attention to certain single prognostic factor and the results remained confused. For the tumour location aspects, the article which mainly paid attention to the prognosis value of the tumour site for different luminal types mainly found tumour site was a more prominent prognosis value for the triple-negative and HER2-enriched groups. The relative good prognosis luminal subgroup (luminal A and luminal B) shared the least article which concerned the value of the tumour location for them. Ours was the first to explore the relationship between the tumour site and the ER positive, HER2 negative subgroup. As for the 
immune markers aspects, papers mainly focused on value of the LMR, the NLR and PLR, and the comparison of the blood marker of the same type. TIL was another prognosis marker which had arrived at some confused conclusions for the ER positive, HER2 negative ones. The ER positive, HER2 negative patients is famous for their good prognosis and uncertain prognosis factors. We have done a breaking study to explore the prognosis of the three uncommon representative variables for the ER positive, HER2 negative breast cancer patients. Our research could help clinicians prescribe more individualized therapies.

We present the following article in accordance with the REMARK reporting checklist (available at http://dx. doi. org/10. 21037/gs-20-622).

\section{Methods}

The first cohort in the present study was comprised of 676 patients who were diagnosed with breast carcinoma from 2010 to 2014, and later underwent NAC. The inclusion criteria included: (I) patients who underwent normal radical or optimized radical mastectomy; (II) patients who had no history of other malignancy; and (III) patients who had not undergone preoperative radiotherapy (RT) or postoperative chemotherapy. The exclusion criteria included (I) Patients with multicentric tumors. (II) (III) The patients without standard bone marrow and impaired hepatic, cardiac, and renal function. (IV) Follow-up information and clinical data was incomplete or missing. Patients with unknown tumor locations the regimens for chemotherapy were: (I) docetaxel and cyclophosphamide (TC; 6 cycles; $n=96,14.2 \%$ ); and (II) doxorubicin and docetaxel (AT; 6 cycles; $\mathrm{n}=270,39.9 \%$ ). The NAC regimens were as follows: AT, doxorubicin $\left(60 \mathrm{mg} / \mathrm{m}^{2}\right)$, and docetaxel $\left(75 \mathrm{mg} / \mathrm{m}^{2}\right)$ by intravenous infusion for 3 weeks for a total of four-to-six cycles; and TC, docetaxel $\left(75 \mathrm{mg} / \mathrm{m}^{2}\right)$, and cyclophosphamide $\left(600 \mathrm{mg} / \mathrm{m}^{2}\right)$ by intravenous infusion for 3 weeks for a total of four-to-six cycles. Most patients diagnosed with tumors in the first clinical stages (IIA, IIB, and IIIA) received AT and TC therapy. The AT regimen was primarily prescribed to patients with stage IIIA, IIIB, and IIIC cancer. After undergoing NAC, all patients underwent surgery with either modified radical mastectomy (MRM) or breastconserving surgery (BCS) with ALN dissection.

All patients underwent a preoperative examination, which included mammography and ultrasonography of the breast and axilla. At the same time, the peripheral blood lymphocyte number and the peripheral blood neutrophil number were recorded for all patients before undergoing NAC. The peripheral blood lymphocyte/peripheral blood neutrophil ratios were calculated. After undergoing surgery, the patients underwent radiation or endocrine therapy. RT was performed using different methods according to the surgical approach, tumor location, and response to NAC, among other factors. In our study, most of the clinical stage II patients who achieved pCR or ypN0 before MRM did not receive any form of post-mastectomy radiation therapy (PMRT). PMRT was strongly recommended to first clinical stage (IIIB-C) patients, regardless of the pathological extent of the disease before surgery, and to patients with clinical stage II with ypN+. For clinical stage IIIa patients, PMRT was administered according to the initial $\mathrm{N}$ stages, luminal subtype, $\mathrm{T}$ stage, age, and the patient's request to undergo treatment. Patients who had undergone BCS were all treated with a 50.4 Gy dose in total, and 1.8 Gy per fraction in the entire breast for five fractions each week. Subsequently, patients received an electron boost (10 Gy) to the target area (tumor bed). For those who underwent MRM, the target area, including the local or nearby lymph nodes and the chest wall, received the same dose. Internal mammary node RT (IMN-RT) was administered to patients based on their clinical or pathological characteristics. IMNRT was applied to treat inner quadrant tumors that might be progressive. According to the National Comprehensive Cancer Network guidelines for adjuvant hormonal therapy, premenopausal females were administered tamoxifen for 5 years, and postmenopausal females were treated with an aromatase inhibitor or with sequential tamoxifen treatment, followed by treatment with an aromatase inhibitor.

The study was a retrospective study itself and was conducted in accordance with the Declaration of Helsinki (as revised in 2013). No ethics committee(s) or institutional review board(s), the number/ID of the approval(s) was available. We enrolled all the patients through our database. The requirement for informed consent was waived.

\section{Hematoxylin-eosin (HE) staining}

Surgically excised breast tumor tissues were immersed in $4 \%$ paraformaldehyde for 4 hours and then transferred to $70 \%$ ethanol. Biopsy samples were cleared in processing cassettes, dehydrated through a gradient series of alcohol solutions, and embedded in paraffin wax blocks. Before immunostaining, 5 - $\mu$ m-thick tissue sections were dewaxed with xylene, rehydrated with decreasing concentrations of ethanol, and washed with phosphate-buffered saline. 


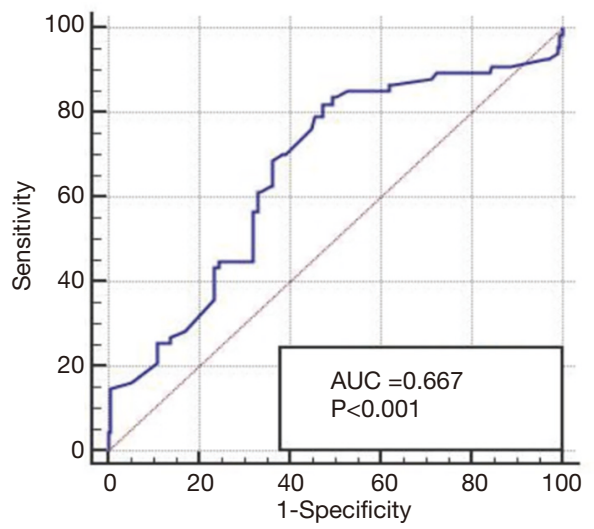

Figure 1 Receiver-operator curve and area under the curve (AUC) for lymphocyte-to-monocyte ratio.

Sections were then stained with HE. After staining, the sections were dehydrated with increasing concentrations of ethanol and xylene.

\section{Evaluation of TIL level}

For patients who underwent HE evaluation of TIL level, we estimated the TIL level within the boundary of invasive cancer. We excluded the adjacent normal tissue, epithelial tissue, massive areas of necrosis in the tumor tissue, hyaline degeneration and fibrosis, and tertiary lymphoid structures around the tumors. Two pathologists evaluated the TIL level based on international guidelines (www. TILsinbreastcancer.org). The TIL level was calculated using the following equation: TIL staining area/total stroma area.

\section{Evaluation of clinical and pathological results}

Patients were further subdivided into five quadrants according to their primary tumor sites after the first physical and imaging examination. It was found that a vast majority of patients had a primary tumor located in the upper-outer quadrant (54\%) and upper-inner quadrant (19.8\%), whereas a minority of patients had a primary tumor in the lowerinner $(13.8 \%)$, central (2.5\%), and lower-outer quadrants $(9.8 \%)$. A larger cohort of patients would be required to improve the quality of our clinical study to address statistical discrepancies. Finally, we excluded the minority and split the cohorts into only three groups according to tumor site: outer quadrant, upper-inner quadrant, and lower-inner/ central quadrant.
The prognostic implications of the pathological findings and treatment features were assessed in addition to the tumor location. Among the pathological and clinical variables, primary tumor size, lymph node status, and HER2 status were assessed as independent variables. Among the treatment characteristics, the types of surgery, IMN-RT, and hormonal therapy were assessed. Furthermore, pCR was defined as no residual invasive tumor in the breast. Tumors with residual intraductal carcinoma were also included in the pCR group.

The clinicopathological features of the different quadrant groups and the different LMR and TIL levels were compared by Pearson's $\chi^{2}$-test. Local recurrence was defined as recurrence that occurred in the ipsilateral breast or the chest wall. Regional recurrence was defined as a relapse found in the ipsilateral axillary, supraclavicular, and/or IMN regions. Recurrence in the contralateral breast, liver, lung, or other distant organs was defined as distant metastasis. Disease-free survival (DFS) refers to the period between the date of any disease recurrence and the date of the first NAC. OS refers to the time from the date of NAC to the date of death. Using the Kaplan-Meier method, we compared the 5-year DFS and OS between the distinct groups. The log-rank test was used for the univariate analysis. Using the Cox proportional hazards regression model, the variables that were statistically significant in the univariate analysis were included in the multivariate analysis. All $\mathrm{P}$ values $<0.05$ indicated statistical significance. For the subgroup analysis, the $\mathrm{P}$ value was corrected according to the Bonferroni correction for reiterated measurement $(\alpha=0.05 / n)$. All statistical analyses were conducted using SPSS version 22.0 software.

\section{$L M R$}

We determined the cut-off value of the LMR based on the receiver operating characteristic curve (ROC) and the area under the curve. As indicated in Figure 1,5.2 was defined as the threshold level for distinguishing between better DFS and worse DFS $(\mathrm{P}<0.001)$. The mean $\mathrm{LMR}$ value was 5.9954, and the range was 1.78-23.70 (Figure 1).

\section{TIL level}

The TIL expression level of 79 patients was analyzed using HE staining; $77.6 \%$ of the 79 patients had a lower expression of TIL $(77.6 \%)$ and $22.4 \%$ had a high expression of TIL (22.4\%). The TIL level range was $3.40-21.30 \%$, and the mean value was $8.4 \%$. We also determined the cut-off 


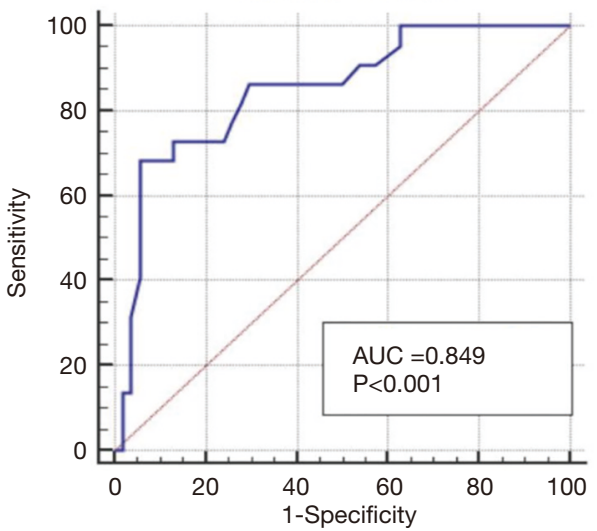

Figure 2 Receiver-operator curve and area under the curve (AUC) for tumor-infiltrating lymphocytes.

value of the TIL based on the ROC curve; 9.9 was defined as threshold level for distinguishing better and worse DFS $(\mathrm{P}<0.001)$. Based on Watanabe et al.'s research (6), $10 \%$ was used as the cut-off level, as it was found that a lower TIL $(\leq 10 \%)$ demonstrated better survival for ERpositive, HER2-negative patients. Their TIL distribution as similar to ours; $70 \%$ of all patients had a lower TIL level. As per previous study (6), we defined the cut-off value as 10\% (Figure 2).

\section{Results}

\section{Patients' clinicopathological features}

A total of 285 patients who underwent NAC in our hospital were enrolled in the present study. Of these, 160 had tumors located at the outer quadrant, 58 had tumors located at the upper-inner quadrant, and 67 had tumors located at the lower-inner/central quadrant. A total of 178 patients had a high expression of LMR. Of these, 107 had a low expression of LMR. The patient and treatment features according to tumor location are listed in Table 1. The patient and treatment features according to tumor sites are listed in Table 2. The ratio of the initial clinical stage II patients was greater for those with higher LMR (41.4\% vs. $34.6 \%, \mathrm{P}=0.015)$. More inner-quadrant patients underwent IMN-RT compared with outer-quadrant ones $(5.1 \% \mathrm{vs}$. $32.8 \%$ vs. $17.9 \%, \mathrm{P}<0.001)$.

\section{Recurrence states}

The total relapse rate was 68 of 285 (23.9\%). The pCR rate was 31 of $285(10.9 \%)$, the rate of complete remission of lymph nodes (ypN0) was $81 / 280(28.9 \%)$, and the whole response (CR + PR) ratio was 241/285 (84.56\%). For all patients, 42 of the 285 patients underwent TC therapy and 243 underwent AT therapy. All patients accepted the whole NAC course; 159 of received the post-mastectomy adjuvant radiation therapy, and 60 received IMN-RT. All of the patients who accepted the BCS received standard PMRT, and 14 of 78 of the patients received IMN-RT. For the patients who accepted the MRM, 129 of 207 received the PMRT, and 46 received IMN-RT.

\section{Univariate analyses}

As indicated in Table 3, pretreatment LMR is correlated with DFS $(\mathrm{P}<0.001$, Figure $3 A)$ and $\mathrm{OS}(\mathrm{P}<0.001$, Figure $3 B)$. Primary tumor site is correlated with the DFS $(\mathrm{P}=0.012$, Figure $4 A)$ and $\mathrm{OS}(\mathrm{P}=0.048$, Figure $4 B)$. The initial clinical stage $(\mathrm{P}<0.001)$, nuclear stage $(\mathrm{P}<0.001)$, luminal subtype $(\mathrm{P}=0.008)$ were correlated with $\mathrm{DFS}$. The luminal subtype $(\mathrm{P}<0.001)$, nuclear grade $(\mathrm{P}<0.001)$, initial clinical stage $(\mathrm{P}=0.028)$ and the ypN0 $(\mathrm{P}=0.031)$ were correlated with the OS.

\section{Multivariate analyses}

Multivariate analysis was applied using the variables that showed statistical significance in the univariate analysis in the Cox proportional hazards regression model. Pretreatment LMR, primary tumor site, nuclear grade, and initial clinical stage were all the independent prognostic factors for DFS $(\mathrm{P}<0.001, \mathrm{P}=0.021, \mathrm{P}=0.016, \mathrm{P}<0.001)$. Pretreatment LMR, luminal subtype, and the nuclear grade were independent prognostic factors for OS $(\mathrm{P}=0.028$, $\mathrm{P}=0.006$, and $\mathrm{P}<0.001$, respectively) (Table 4).

\section{Clinicopathological features of patients who underwent} TIL

In total, 79 patients underwent TIL examination. The TIL level range was $3.40-21.30 \%$, and the mean value was $8.4 \%$, using $10 \%$ as the cut-off value. The correlation between the clinicopathological features and the TIL level are shown in Table 5 .

\section{Univariate analyses}

In the subgroup of patients who underwent TIL evaluation, 
Table 1 Patient and treatment features according to LMR level ( $\mathrm{n}=285)$

\begin{tabular}{|c|c|c|c|}
\hline Variables & Pretreatment LMR (>5.2), n (\%) & Pretreatment LMR ( $\leq 5.2), \mathrm{n}(\%)$ & $P$ value \\
\hline Outer & $99(55.6)$ & $61(57.0)$ & \\
\hline Upper-inner & $35(19.7)$ & $23(21.5)$ & \\
\hline Lower-inner/central & $44(24.7)$ & $23(21.5)$ & \\
\hline I & $20(11.2)$ & $10(9.3)$ & \\
\hline II & $151(84.8)$ & $85(79.4)$ & \\
\hline III & $7(4.0)$ & $12(11.3)$ & \\
\hline PCR or not & & & 0.053 \\
\hline Surgery & & & 0.844 \\
\hline BCS & $48(27.0)$ & $30(29.2)$ & \\
\hline MRM & $130(73.0)$ & $77(70.8)$ & \\
\hline Clinical stage & & & 0.015 \\
\hline II & $81(45.5)$ & $37(34.6)$ & \\
\hline III & $97(54.5)$ & $70(65.4)$ & \\
\hline RT & & & 0.864 \\
\hline Yes & $100(56.2)$ & $59(55.1)$ & \\
\hline Yes & $35(19.7)$ & $22(20.6)$ & \\
\hline No & $143(80.3)$ & $85(79.4)$ & \\
\hline Standard endocrine therapy & & & 0.897 \\
\hline Yes & $131(73.6)$ & $78(72.9)$ & \\
\hline No & $47(26.4)$ & $29(27.1)$ & \\
\hline Luminal subtype & & & 0.307 \\
\hline Luminal A & $73(41.0)$ & 37 (34.6) & \\
\hline Luminal B & $105(59.0)$ & $70(65.4)$ & \\
\hline \multicolumn{4}{|l|}{ Resection margin } \\
\hline Negative & $164(92.1)$ & $97(90.7)$ & 0.646 \\
\hline Positive & $14(7.9)$ & $10(9.3)$ & \\
\hline
\end{tabular}

Table 1 (continued) 
Table 1 (continued)

\begin{tabular}{lcc}
\hline Variables & Pretreatment LMR $(>5.2), \mathrm{n}(\%)$ & Pretreatment LMR $(\leq 5.2), \mathrm{n}(\%)$ \\
\hline PR & $103(57.9)$ & $58(54.2)$ \\
Negative & $75(42.1)$ & $49(45.8)$ \\
Positive & & \\
Pathological stage & $136(76.4)$ & $84(78.5)$ \\
0-II & $42(23.6)$ & $23(21.5)$ \\
III & & 0.682 \\
Age & $59(33.1)$ & $41(38.3)$ \\
$>50$ & $119(66.9)$ & $66(61.7)$ \\
\hline 50 & & 0.376 \\
\hline
\end{tabular}

AT, Doxorubicin Docetaxel BCS, breast-conserving surgery; IMN-RT, internal mammary node radiotherapy; LMR, lymphocyte-to-monocyte ratio; MRM, modified radical mastectomy; NAC, neoadjuvant chemotherapy; $\mathrm{PCR}$, pathological complete response; PR, progesterone receptor; $\mathrm{RT}$, radiation therapy; $\mathrm{TC}$, docetaxel cyclophosphamide.

endocrine therapy $(\mathrm{P}=0.0110)$, pathological stage $(P=0.0167)$, initial clinical stage $(\mathrm{P}<0.001)$, nuclear grade $(\mathrm{P}=0.0136)$, and TIL level $(\mathrm{P}=0.010)$ were correlated with DFS (Figure $5 A)$. Initial clinical stage $(\mathrm{P}<0.001)$ and ypN0 $(\mathrm{P}=0.0232)$ were prognostic factors for OS (Figure $5 B$ and Table 6).

\section{Multivariate analysis}

Initial clinical stage $(\mathrm{P}<0.001)$ and TIL level $(\mathrm{P}=0.027)$ were correlated with DFS; initial clinical stage $(\mathrm{P}<0.001)$ was the only independent prognostic factors for OS (Table 7).

\section{TIL level in breast cancer tissue}

We evaluated the TIL level in postoperative breast cancer tissue using HE staining. As shown in Figure 6, the TIL level of 63 of $79(79.7 \%)$ patients was $\leq 10 \%$. The expression of the remaining $16(21.3 \%)$ was $>10 \%$.

\section{Discussion}

In the present study, we explored the prognostic significance of the tumor site, TIL level, and pretreatment LMR for ER-positive, HER2-negative patients. According to the results of multivariate analyses, the tumor location was an independent prognosis for 5-year DFS $(\mathrm{P}=0.021)$. Pretreatment LMR was associated with both 5 -year DFS and $\mathrm{OS}(\mathrm{P}<0.001$ and $\mathrm{P}<0.001$, respectively). Unlike previous studies, our study described the prognostic significance of three uncommon factors (primary tumor location, LMR, and TIL). As the ER-positive, HER2negative subgroup seldom shares the same prognostic factors (i.e., pCR) as other luminal types, finding a suitable prognostic marker remains a challenge for clinicians. Therefore, in the present study we aimed to discover a more precise prognosis for ER-positive, HER2-negative tumors. Effective remedies, such as PMRT or IMN-RT, should be provided based on relapse risk factors, which are based on the adverse prognosis. For IMN-RT, after a long course of NAC, surgery, and adjuvant radiation therapy, IMN-RT is difficult to achieve in terms of technology and compliance limitations. Implementing IMN-RT to patients remains controversial. It is important to explore whether PMRT may also benefit breast cancer patients through residual lymph node reduction mechanisms. Therefore, more accurate predictive biomarkers will be critical for personalizing ER-positive, HER2-negative patient treatments in the clinical setting in the future.

With regard to the tumor location aspect, we found that the lower-inner and central quadrants shared the worst survival benefit. Our study mainly investigated the ERpositive, HER2-negative subtype, the prognosis of which is better than those of other luminal types. There have been several previous retrospective studies focusing on the prognosis of the tumor location with regard to certain tumor subtype. In their retrospective studies, Wu et al. found that primary tumor location in the LIQ was an independent 
Table 2 Patient and treatment features according to different tumor locations ( $\mathrm{n}=285)$

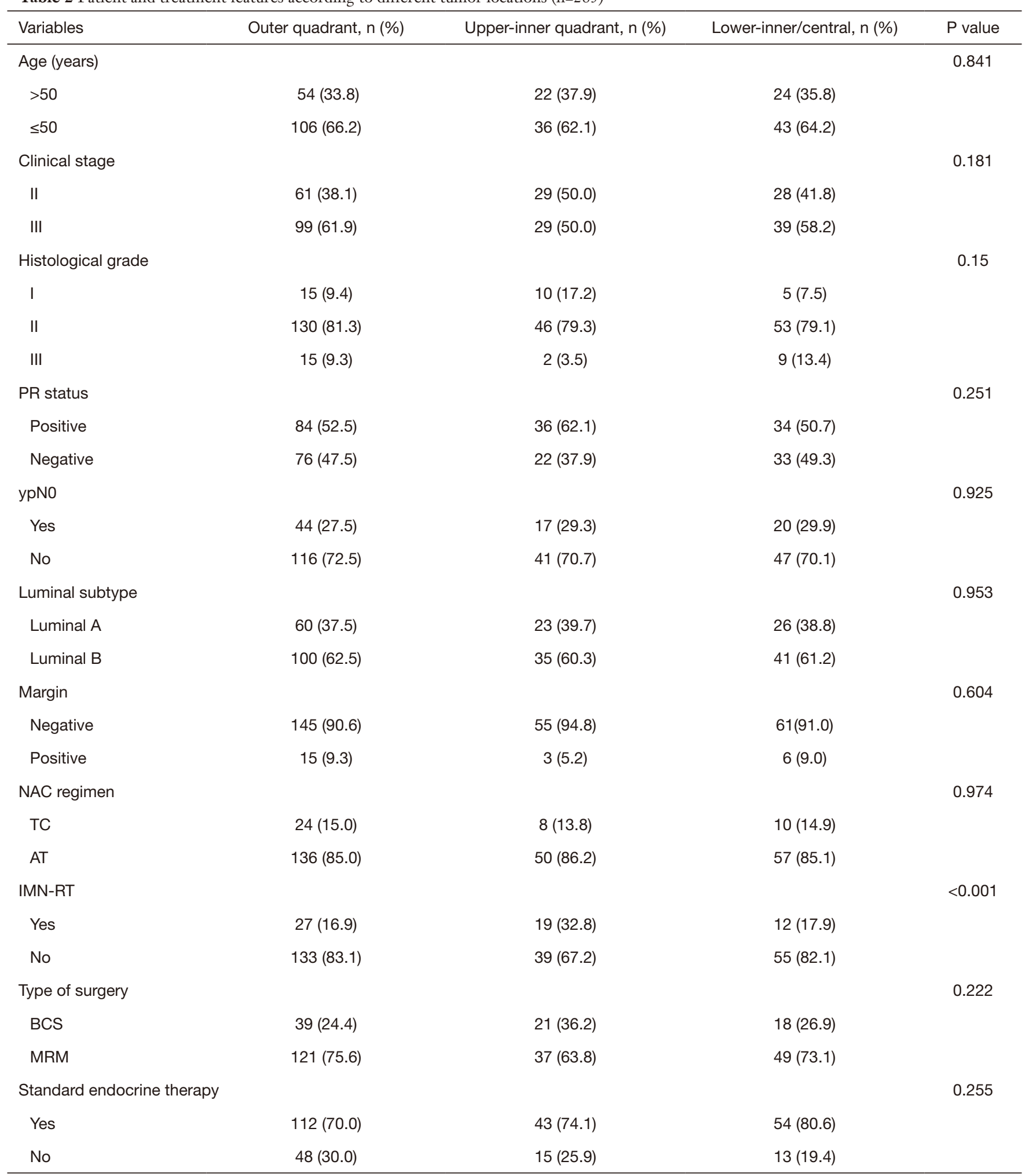

Table 2 (continued) 
Table 2 (continued)

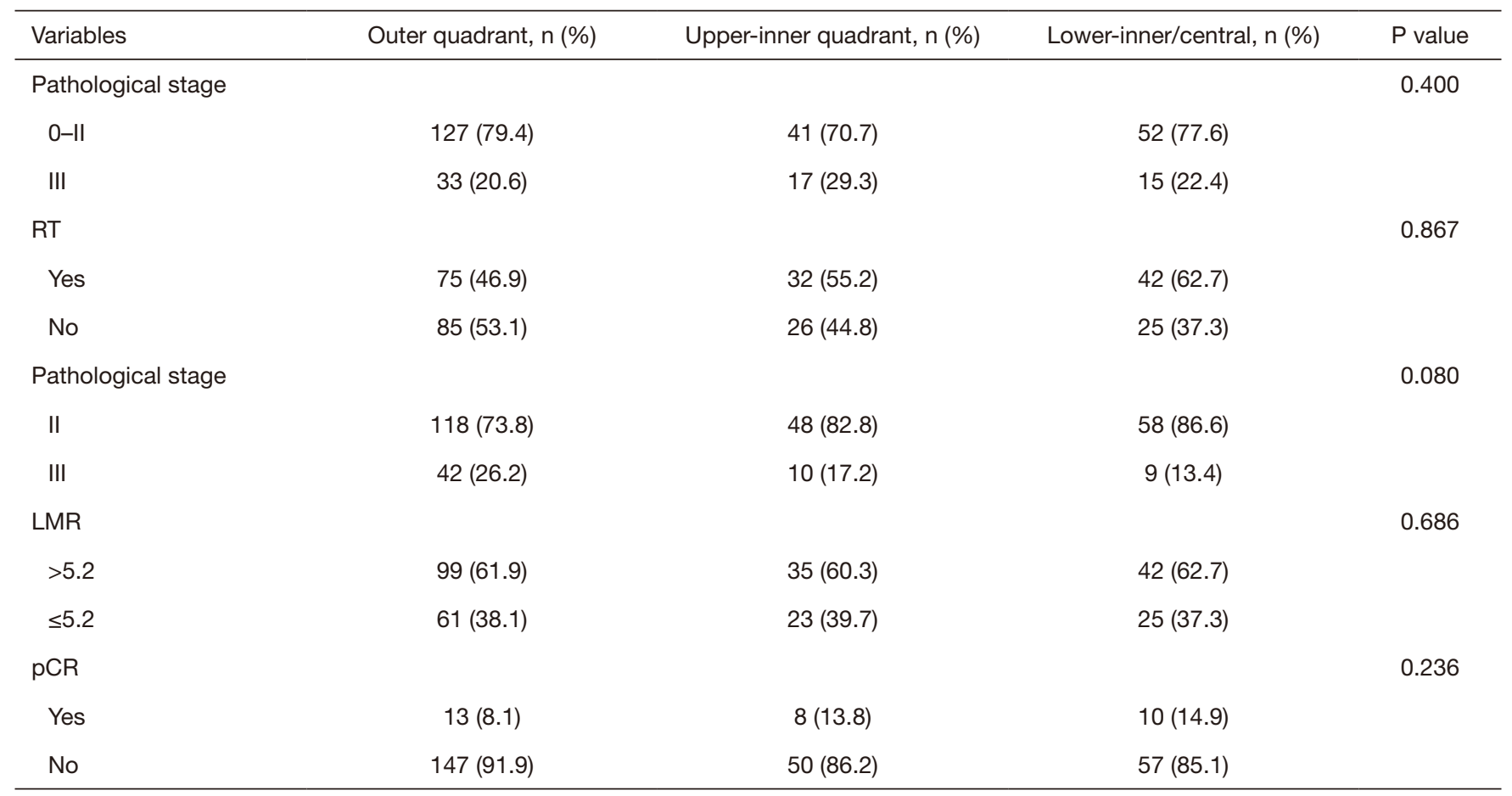

AT, doxorubicin docetaxel; BCS, breast-conserving surgery; IMN-RT, internal mammary node radiotherapy; LMR, lymphocyte-to-monocyte ratio; MRM, modified radical mastectomy, NAC, neoadjuvant chemotherapy; pCR, pathological complete response; PR, progesterone receptor; RT, radiation therapy; TC, docetaxel cyclophosphamide; ypNO, N0 after neoadjuvant chemotherapy.

poor prognostic factor for the T1-2N0M0 triple-negative breast cancer (7). After comprehensively comparing this with other prognostic indexes, they suggested that the inner quadrant was associated with the poor LRR (localregional recurrence) and LRRFS (local-regional recurrence free survival) for early breast cancer patients with one-tothree positive axillary lymph nodes (7). The retrospective study conducted by Niu et al. raised the same concerns: Their finding indicated that lower-inner quadrant patients shared the worst prognosis of survival for the early-stage breast cancer patients (8). The aim of the study conducted by Yang et al. was to explore the prognosis of the primary tumor site; they concluded that tumors located in the LIZ was an independent prognostic factor for poor survival of early breast cancer (9). Most of the previously published retrospective studies focusing on clinical early-stage breast cancer have determined that the lower-inner quadrant and central quadrant share the worst prognosis: the highest rate of IMN metastasis $(10,11)$. With regard to the luminal subtype, Lim et al. found that the poor prognosis of the lower-inner quadrant was more prominent in the HER2- enriched, triple-negative subgroup (12). A few studies have focused on the impact of the tumor site for the different luminal subtypes $(8,13,14)$. However, our study was the first to explore the prognostic significance of the tumor site for the ER-positive, HER2-negative patients. As these patients have a lower relapse rate, and original clinicopathological factors seldom play a predictive role, with regard to the variable selection, we took the primary tumor location into consideration. The inner quadrant always has poor survival prognosis in the HER2-enriched, triple-negative subgroup. In the present study, we found that the inner lower-inner and central quadrants shared the worst survival prognosis for the ER-positive, HER2-negative patients. This finding indicates that the primary tumor site is an independent prognostic factor for these patients.

The mechanism underlying the poor prognosis of the inner-quadrant tumors has not been properly understood, and most many researchers believe that the hidden inner mammary lymph nodes are correlated with poor prognosis $(2,15,16)$. The only feasible treatment for residual IMN metastasis is IMN-RT. In their study, Yang et al. concluded 
Table 3 Clinicopathological variables for 5 -year disease-free, locoregional recurrence-free, distant metastasis-free, and overall survival in the univariate analysis

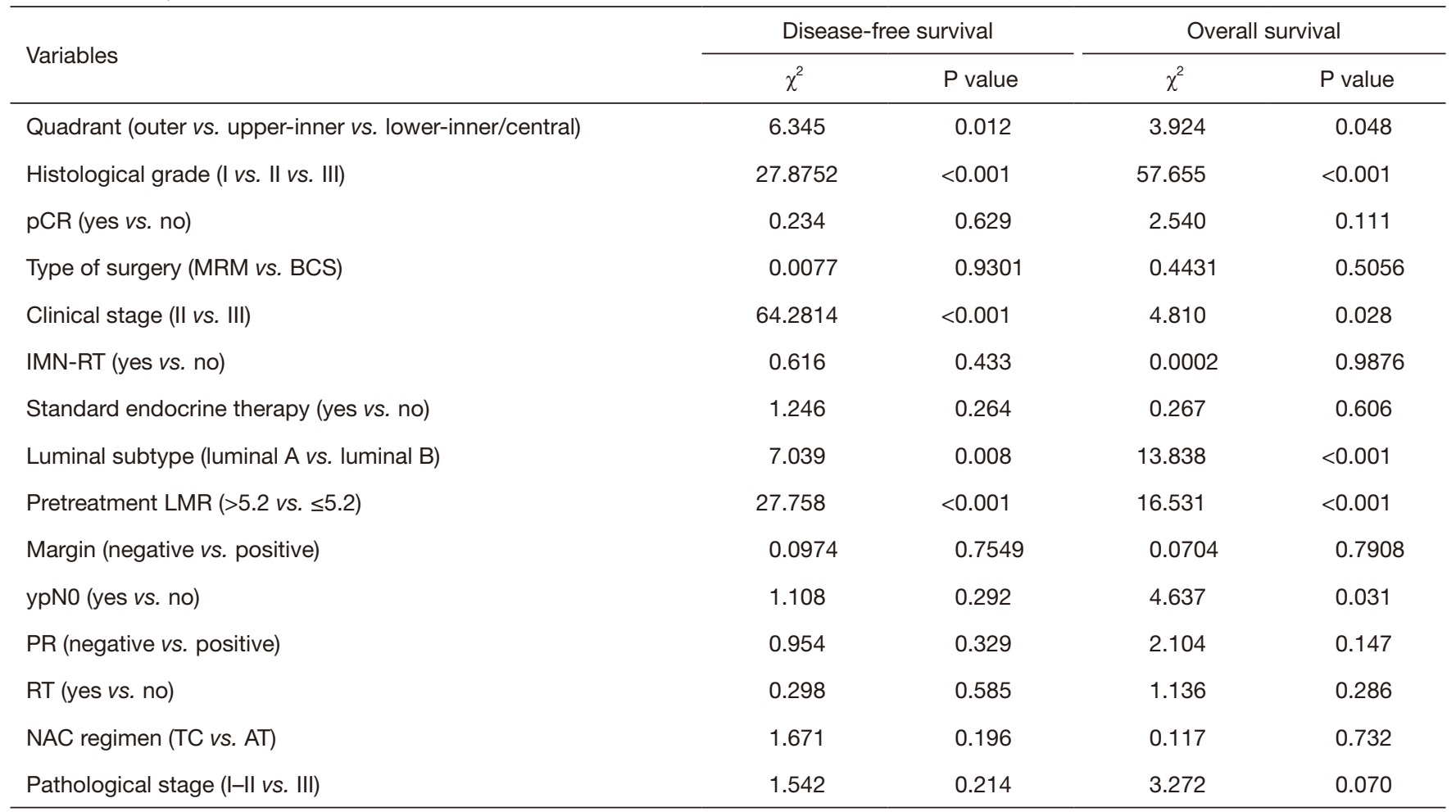

AT, doxorubicin docetaxel; BCS, breast-conserving surgery; IMN-RT, internal mammary node radiotherapy; LMR, lymphocyte-to-monocyte ratio; MRM, modified radical mastectomy, NAC, neoadjuvant chemotherapy; $\mathrm{PCR}$, pathological complete response; PR, progesterone receptor; RT, Radiation Therapy; TC, docetaxel cyclophosphamide; ypNO, NO after neoadjuvant chemotherapy.
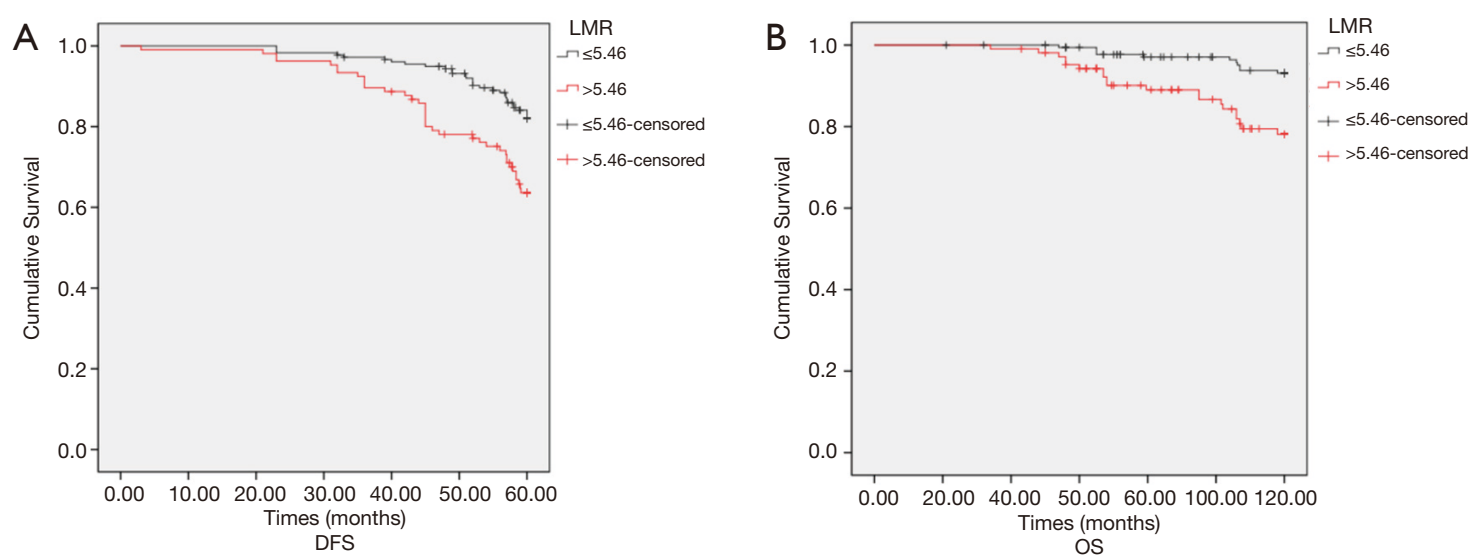

Figure 3 Survival curves for patients stratified according to lymphocyte-to-monocyte ratio. (A) Five-year disease-free survival (DFS); (B) overall survival (OS).

that the IMN-RT was particularly suitable for patients with larger IMN (17). In their study, Aleknavičius et al. found that IMN-RT was correlated with a better survival outcome for patients with moderate-risk disease (stage
T1-2N1), and showed no association for better survival for high-risk disease (stage T3-4N2-3) (18). In their study, Kim et al. reported that IMN-RT was not associated with DFS in IMN patients (19). The result of our study was 

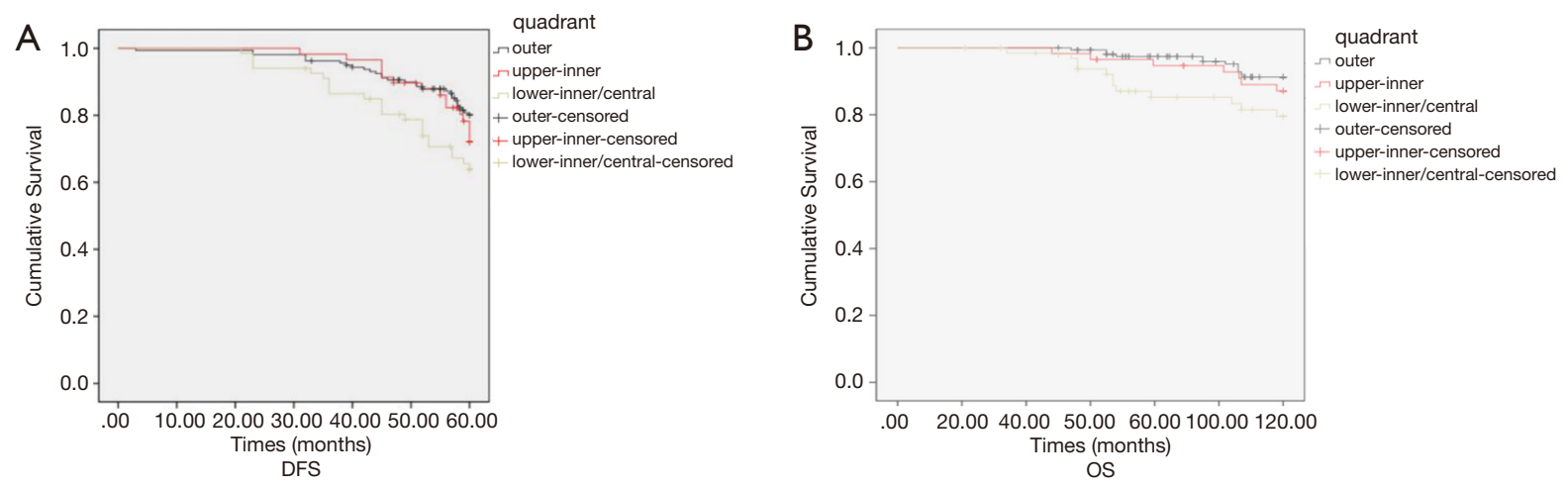

Figure 4 Survival curves according to tumor location. (A) Five-year disease-free survival (DFS); (B) overall survival (OS).

Table 4 Clinicopathological variables for 5-year disease-free and overall survival in the multivariate analysis

\begin{tabular}{|c|c|c|c|c|c|}
\hline Clinicopathological variables & $\beta$ & SE & $P$ value & Hazards ratio & $95 \%$ confidence interval \\
\hline Quadrant (outer and upper-inner vs. lower-inner/central) & 0.626 & 0.272 & 0.021 & 1.870 & $1.099-3.185$ \\
\hline Pretreatment LMR (>5.2 vs. $\leq 5.2)$ & 1.007 & 0.267 & $<0.001$ & 2.737 & $1.623-4.618$ \\
\hline Histological grade (I-II vs. III) & 0.845 & 0.352 & 0.016 & 2.329 & $1.168-4.642$ \\
\hline \multicolumn{6}{|l|}{ 5-year overall survival } \\
\hline Luminal subtype (luminal A vs. luminal B) & 1.688 & 0.616 & 0.006 & 5.407 & $1.616-18.088$ \\
\hline Pretreatment LMR (>5.2 vs. $\leq 5.2)$ & 0.932 & 0.423 & 0.028 & 2.539 & $1.108-5.819$ \\
\hline Histological grade (I-II vs. III) & 1.790 & 0.443 & $<0.001$ & 5.987 & $2.513-14.263$ \\
\hline
\end{tabular}

In the multivariate analysis, only variables with $\mathrm{P}<0.1$ and other meaningful variables in the univariate analysis were included. $\mathrm{LMR}$, lymphocyte-to-monocyte ratio; SE, standard error.

largely consistent with the findings of the abovementioned clinical retrospective studies; inferior results of the innerquadrant tumors were more prominent for patients with a better survival prognosis. Due to the low relapse rate, once there is recurrence in this subgroup of patients, clinicians will have the opportunity to discover the relapse risk factor more precisely. That step is the breakthrough point for our retrospective study. However, conflicting opinions on the benefit of IMN-RT exists; for example, cardiac toxicity is considered a side-effect of IMN-RT (20). After assessing 2,126 patients who underwent the IMN-RT in their cohort, Dess et al. found that there was no statistically significant association between IMN-RT and ICE (Ischemic Cardiac Events) (21). In another study on the cardiotoxicity of IMN-RT, it was concluded that the risk of radiation- induced cardiotoxicity must be weighed individually (22). However, in our study, IMN-RT did not show a survival benefit for patients because our IMN-RT rate was not high enough to make a conclusion. Only a small proportion of inner-quadrant tumors $(24.8 \%)$ underwent both the postsurgery RT and the IMN-RT. The clinical benefit of IMNRT for the inner-quadrant tumors requires further studies with more patients. Our results indicated that, for the good prognostic subgroup patients, the primary tumor site located at the lower-inner/central quadrant means a higher rate of relapse and that follow-up and further treatment are required.

The prognostic significance of LMR has been proved by many types of malignant tumors. In their study, Zhou et al. investigated the predictive significance of the LMR 
Table 5 Patient and treatment features according to TIL ( $\mathrm{n}=79$ )

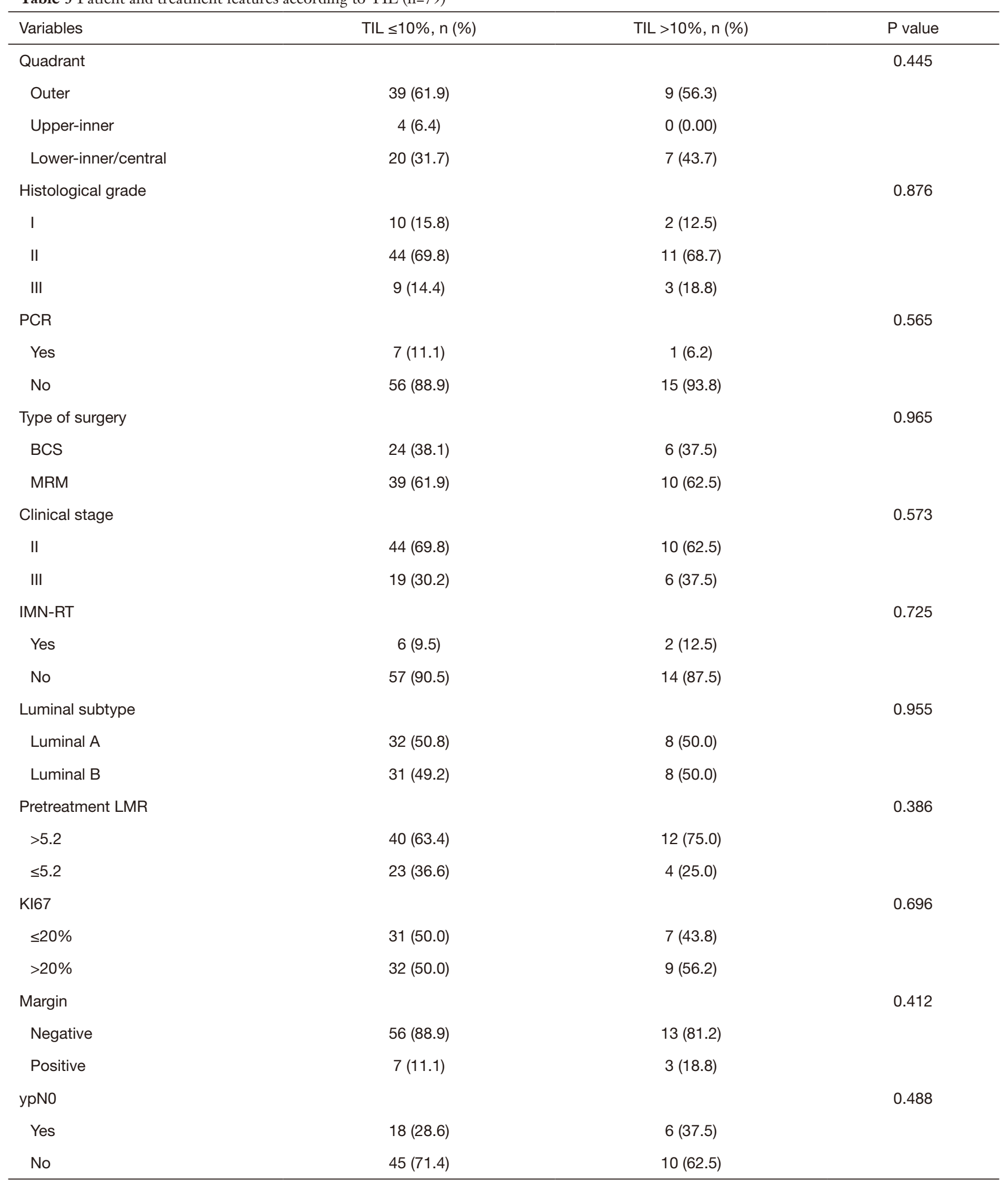

Table 5 (continued) 
Table 5 (continued)

\begin{tabular}{|c|c|c|c|}
\hline Variables & TIL $\leq 10 \%, n(\%)$ & TIL > 10\%, n (\%) & $P$ value \\
\hline Negative & $12(19.0)$ & $1(6.2)$ & \\
\hline Positive & $51(81.0)$ & 15 (93.8) & \\
\hline RT & & & 0.198 \\
\hline No & $31(49.2)$ & 5 (31.2) & \\
\hline Pathological stage & & & 0.272 \\
\hline $0-I I$ & $47(74.6)$ & $14(87.5)$ & \\
\hline III & $16(25.4)$ & $2(12.5)$ & \\
\hline No & $14(25.0)$ & $6(37.5)$ & \\
\hline
\end{tabular}

BCS, breast-conserving surgery; IMN-RT, internal mammary node radiotherapy; LMR, lymphocyte-to-monocyte ratio; MRM, modified radical mastectomy, pCR, pathological complete response; PR, progesterone receptor; TIL, tumor-infiltrating lymphocytes; ypN0, N0 after neoadjuvant chemotherapy.
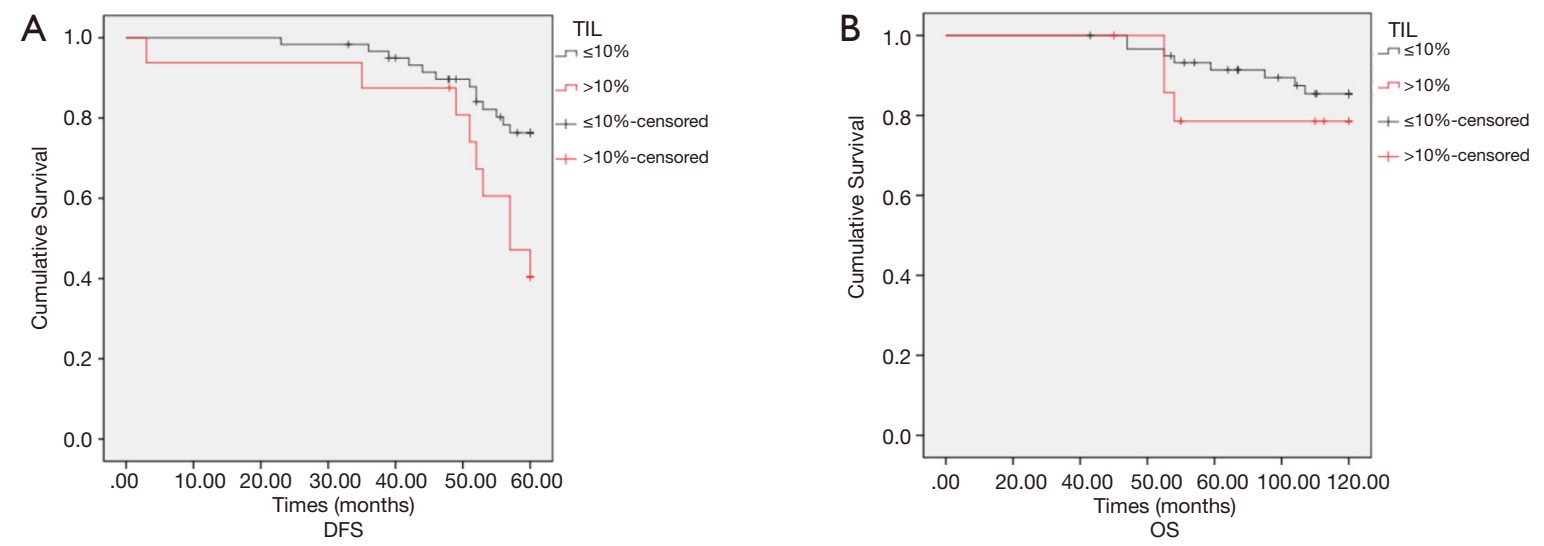

Figure 5 Survival curves according to tumor-infiltrating lymphocytes (TIL). (A) Five-year disease-free survival (DFS); (B) overall survival (OS).

and the lymphocyte-neutrophil ratio. Their research revealed that the LMR is recognized as the independent prognostic factor for the RFS (recurrence free survival), and the NLR (neutrophils lymphocyte ratio) is correlated with OS (23). The results of the multivariate analysis showed that the LMR also played an important role in predicting the survival prognosis for PTC (papillary thyroid carcinoma) patients (24). Our retrospective study indicated that LMR is the independent prognostic factor for ER-positive, HER2negative patients. In their study, Huszno and Kolosza compared the value of the most common three peripheral blood immune prognostic factors: the NLR, PLR (platelet lymphocyte ratio), and LMR. The result showed that the NLR and PLR were the independent prognostic factors for breast cancer patients, whereas the LMR was not the valuable prognosis in the multivariate analysis (25). In their retrospective analysis on locally advanced breast cancer, $\mathrm{Ni}$ et al. stated that an LMR $>4.25$ was associated with a good DFS prognosis, after separately considering lymphocyte counts and the peripheral blood monocyte counts. They 
Table 6 Clinicopathological variables for 5-year disease-free and overall survival in the univariate analysis

\begin{tabular}{|c|c|c|c|c|}
\hline Variables & \multicolumn{2}{|c|}{ Disease-free survival } & \multicolumn{2}{|c|}{ Overall survival } \\
\hline Standard endocrine therapy (yes vs. no) & 6.4661 & 0.0110 & 1.0993 & 0.2944 \\
\hline Luminal subtype (luminal A vs. luminal B) & 2.3241 & 0.1274 & 3.6774 & 0.0552 \\
\hline Clinical stage (II vs. III) & 39.7845 & $<0.001$ & 16.9809 & $<0.001$ \\
\hline Pathological stage (0-II vs. III) & 5.7243 & 0.0167 & 1.6589 & 0.1978 \\
\hline Histological stage (I-I II vs. III) & 6.0889 & 0.0136 & 2.862 & 0.091 \\
\hline ypN0 (yes vs. no) & 0.6955 & 0.4043 & 5.1562 & 0.0232 \\
\hline TIL ( $\leq 10 \%$ vs. $>10 \%)$ & 6.7047 & 0.010 & 0.6489 & 0.4205 \\
\hline
\end{tabular}

LMR, lymphocyte-to-monocyte ratio; TIL, tumor-infiltrating lymphocytes; ypNO, NO after neoadjuvant chemotherapy.

Table 7 Clinicopathological variables for 5-year disease-free and overall survival in the multivariate analysis

\begin{tabular}{|c|c|c|c|c|c|}
\hline Clinicopathological variables & $\beta$ & SE & $P$ value & Hazards ratio & $95 \%$ confidence interval \\
\hline Clinical stage (II vs. III) & 2.172 & 0.558 & $<0.001$ & 8.777 & $2.939-26.211$ \\
\hline TIL ( $\leq 10 \%$ vs. $>10 \%)$ & 1.165 & 0.528 & 0.027 & 3.207 & $1.140-9.018$ \\
\hline \multicolumn{6}{|l|}{ 5-year overall survival } \\
\hline Histological grade (I-II vs. III) & 0.687 & 0.630 & 0.276 & 1.988 & $0.578-6.840$ \\
\hline
\end{tabular}

In the multivariate analysis, only variables with $\mathrm{P}<0.1$ and other meaningful variables in the univariate analysis were included. SE, standard error.
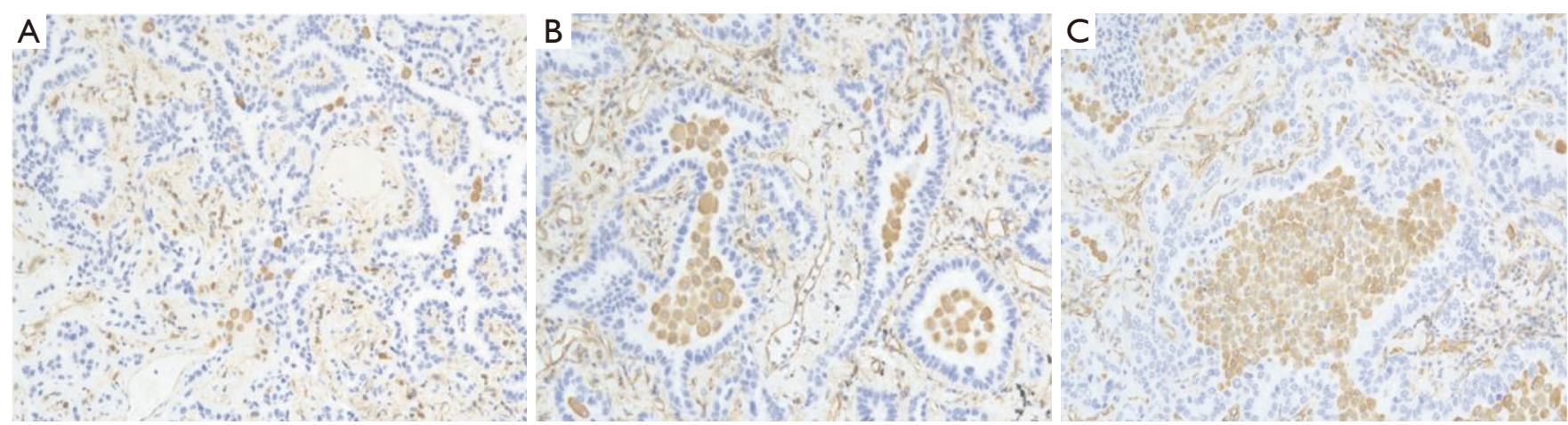

Figure 6 Representative hematoxylin-eosin staining of the tumor-infiltrating lymphocytes (TIL) of breast cancer patients (200x). (A) TIL expression: 4\%; (B) TIL expression: $12 \%$; (C) TIL expression: $20 \%$.

found that the lymphocyte count was positively correlated with DFS, whereas the peripheral blood monocyte count was the opposite (26). In another study on the predictive value of the LMR for luminal breast cancer patients, it was acknowledged that the LMR was an independent prognostic factor for luminal patients. The LMR value $(\geq 5.2)$ indicated 
a good survival prognosis (27). Moreover, in another study that focused on the predictive value of the LMR on triplenegative breast cancer patients, the authors concluded that low LMR levels result in worse DFS for these patients. However, those results were not consistent with other studies. Jia et al. found that higher LMR levels indicate a higher probability of relapse for triple-negative breast cancer patients; however, this result was no longer true after the multivariate analysis. The author explained that their study is the first study to evaluate the prognostic value of both the NLR and the LMR in breast cancer subtypes and they found NLR was superior to LMR. Further validation studies were required to confirm the result (28). Similar to the tumor location aspects, there have been few studies concerning the prognostic value of the LMR to luminal patients, demonstrating lower influence of the immune system on the luminal $(29,30)$. Our finding corroborates those of other studies; chemotherapy drugs can increase the killing effect of immune cells on cancer cells by enhancing the antigen presentation of $\mathrm{T}$ lymphocytes. Conversely, monocytes play an important role in inflammatory response, and a high gene expression indicates a greater risk of metastasis. Our research further explored the prognostic value of the LMR for ER-positive, HER2-negative patients. However, the results need to be confirmed further.

The LMR has been recognized as a valuable prognostic factor for the ER-positive, HER2-negative subgroup. When it turns to the other two biomarkers: the NLR and the PLR.A study of 1,570 Chinese breast cancer patients found that neither NLR nor PLR was significantly associated with outcome in any of the other breast cancer subtypes expect the triple-negative ones (31). In fact, several studies have found a differential impact for PLR or NLR according to breast cancer subtype $(32,33)$. However, the impact of the NLR and PLR (especially the PLR) were more confused than LMR for the ER positive, HER2 negative patients: fewer attention was paid for the prognosis value of the PLR for the ER positive, HER2 negative patients. NLR played an important role in predicting the PCR in several articles. One of them found that NLR had a statistically significant relationship with the probability of PCR for luminal B subtype. While for the ER positive HER2 negative ones, PCR was not an independent feature for a better survival $(34,35)$. After systematical and thorough analysis, maybe LMR is more suitable for the ER positive, HER2 negative subgroup. The main difficulty for our study was deciding the optimal cut-off value. We did not choose the average or the median for fear of the high randomness and complexity of the data. When we applied the ROC curve to determine the cut-off value, we faced the same dilemma of which is best suited: the DFS or the OS? The OS is not a better choice, and accepting it may lead to more difficulty in the statistical analysis. Finally, we chose the DFS to determine the proper cut-off for the LMR and it successfully predicted both the DFS and OS.

Immune microenvironments are closely associated with the development of cancer, although most researchers state that the development of the tumor is correlated with immune escape. However, it is surprising that the value and function of antitumor immunity cannot be replaced, even at advanced cancer stages. TIL represent the immune status of patients, and an increasing number of studies have endogenously explored the prognostic significance of the LMR. For example, Dieci et al. found that every $10 \%$ increase in TIL after NAC always results in a $21 \%$ decrease in the risk of death. However, little research has focused on this, and there is still uncertainty regarding the value of post-NAC TIL and the significance of TIL for luminal tumors (36).

In their study, Hwang et al. found that among HER2positive patients who underwent TCHP (Docetaxel, carboplatin, Herceptin therapy), a higher pretreatment TIL was correlated with pCR, and post-treatment TIL indicated longer survival benefit (37). The aim of Watanabe et al.'s (6) study was to explore the prognostic value of post-NAC TIL. In their exploration of different luminal subtypes, the result between the ER-positive, HER2-negative group and the HER2-enriched/triple-negative subgroup was diametrically opposite. One crucial difference was that a low post-treatment TIL level correlated with a better survival for the ER-positive, HER2-negative patients. Hamy et al. found that HER2-enriched patients with higher levels of TIL correlated with a worse DFS and higher probability of metastasis (38).

Our clinical trial found that lower post-NAC is a good independent prognosis for DFS; however, it was not correlated with OS. The mechanism behind the result is complex. In their study, Hamy et al. explained that higher TIL was correlated with higher tumor mutation load and mitotic index. Watanabe et al. found that the higher the mutation burden, the worse the survival prognosis (6). They further reported that after a comprehensive course of therapies, higher TIL indicated higher immune activity and higher residual tumor burden, which implies a worse prognosis. In their study, Denkert et al. found that, for the HER2-enriched and the triple-negative subgroup, 
numerous immune indices are responsible for the prognosis (i.e., T lymph cells, B lymph cells, and macrophages), whereas with regard to luminal subtype aspects, most of immune indices have only a slight prognostic impact, with the exception of B lymph cells and myeloid dendritic cells . However, this phenomenon was unclear for TIL level, which is impacted not only by the immune system but also by the characteristics of the breast cancer itself. Denkert et al. found that a high TIL level is correlated with better survival for luminal patients with histological stage III. However, with regard to the different ki-67 level subgroup, they did not find a correlation between TIL and prognosis (39). ER plays a key role in the formation of the microenvironment of the luminal tumor, thus the ER of the cancer could render immune response immediate by Th2 cells and decrease the MHC-II (major histocompatibility complex-II) expression. Any of the these findings may have led to the decrease of the $\mathrm{T}$ lymph nodes.

The value of post-NAC TIL is mainly discussed in the HER2-enriched and triple-negative subgroups. As a result of these findings, we examined the value of TIL for the luminal subtype. Similarly, in the luminal patients, studies exploring the value of TIL were rare; none reached an appropriate conclusion, and none shared the same design. Our clinical trial only focused on the TIL level of 79 patients. The main obstacle of our study was the distribution bias of the TIL level; only approximately $21.1 \%$ of our cohort had a high TIL level. As the biased distribution of the TIL level was similar to that of a larger study cohort, we were able to reach a positive conclusion by using our cohort of patients. More high-quality and precise data are needed to further confirm our results.

Although the tumor location, LMR, and TIL level all showed a strong prognostic value for ER-positive, HER2negative patients, to overcome the relapse rate for this subgroup of patients, it is important to find a more accurate prognostic factor and new therapies. In addition, the majority of current studies are trying to explore alternative therapies for luminal patients. Prolonging endocrine therapy has been suggested. The clinical trial enrolled 1,912 HR (Hormone Receptor) -positive patients. All patients underwent Tamoxifen therapy for 2-3 years, and the trial compared the efficacy of prolonged 3-year versus 6-year anastrozole therapy. It was found that prolonging endocrine therapy could prolong the survival benefit only in the ER/ PR (Progesterone Receptor)-positive, HER2-negative, high tumor burden, and post-AC (adjuvant chemotherapy) therapy subgroup. When analyzing the patients as a whole, prolonging anastrozole did not show a prognostic benefit. The researchers plan to extend endocrine therapy to 9 years in the future (40). It will be important to explore whether improving radiation strategies may have a positive influence on patient survival through the removal of residual lesion mechanisms. As discussed earlier, the significance of IMNRT on inner-quadrant tumors is unclear. Debate also exists in the in the area of PMRT for ER-positive, HER2-negative patients. In their study, Wei found that PMRT could result in survival benefits for T1-2N1 luminal A patients (41).

Researchers have found that accepting standard PMRT does not result in relapse-free survival for triple-negative patients. In contrast, the clinical value is limited for the HR-positive patients, even in non-pCR situations. More research on ER-positive, HER2-negative patients is needed to explain this mechanism, and further studies are needed to tackle the high rate of relapse observed in the ER-positive, HER2-negative subgroup.

\section{Limitations of the study}

As with other studies, the present study has several limitations. Bräutigam et al. found that outer tumors had a better survival rate than the inner ones. However, the limitation of their research was obvious: the patients were mainly recruited from 1984 to 1995 . The distribution of the clinicopathological factors between the inner and outer quadrants differed significantly (42). We found that, the earlier the study was, the more difficult it was to achieve a reasonable result. To the best of our knowledge, the present study was also the first to explore three uncommon prognostic factors for a good prognostic subgroup of patients: ER-positive, HER2-negative patients. We attempted to make the clinical data as reliable as possible by excluding all patients with incomplete information. We allocated our patients into specific groups, which enabled us to determine that the primary tumor site, LMR, and TIL level are independent prognostic factors for ER-positive, HER2-negative patients.

\section{Acknowledgments}

Funding: None.

\section{Footnote}

Reporting Checklist: The authors have completed the REMARK reporting checklist. Available at http://dx.doi. 
org/10.21037/gs-20-622

Data Sharing Statement: Available at http://dx. doi. org/10. 21037/gs-20-622

Conflicts of Interest: Both authors have completed the ICMJE uniform disclosure form (available at http://dx. doi. org/10. 21037/gs-20-622). The authors have no conflicts of interest to declare.

Ethical Statement: The authors are accountable for all aspects of the work in ensuring that questions related to the accuracy or integrity of any part of the work are appropriately investigated and resolved. The study was a retrospective study itself and was conducted in accordance with the Declaration of Helsinki (as revised in 2013). No ethics committee(s) or institutional review board(s), the number/ID of the approval(s) was available. We enrolled all the patients through our database. The requirement for informed consent was waived.

Open Access Statement: This is an Open Access article distributed in accordance with the Creative Commons Attribution-NonCommercial-NoDerivs 4.0 International License (CC BY-NC-ND 4.0), which permits the noncommercial replication and distribution of the article with the strict proviso that no changes or edits are made and the original work is properly cited (including links to both the formal publication through the relevant DOI and the license). See: https://creativecommons.org/licenses/by-nc-nd/4.0/.

\section{References}

1. Ji F, Xiao WK, Yang CQ, et al. Tumor Location of the Central and Nipple Portion Is Associated With Impaired Survival for Women With Breast Cancer. Cancer Manag Res 2019;11:2915-25.

2. Lohrisch C, Jackson J, Jones A, et al. Relationship between tumor location and relapse in 6,781 women with early invasive breast cancer. J Clin Oncol 2000;18:2828-35.

3. Park C, Seid P, Morita E, et al. Internal mammary sentinel lymph node mapping for invasive breast cancer: implications for staging and treatment. Breast J 2005;11:29-33.

4. Peng Y, Chen R, Qu F, et al. Low pretreatment lymphocyte/ monocyte ratio is associated with the better efficacy of neoadjuvant chemotherapy in breast cancer patients. Cancer Biol Ther 2020;21:189-96.
5. Loi S, Sirtaine N, Piette F, et al. Prognostic and predictive value of tumor-infiltrating lymphocytes in a phase III randomized adjuvant breast cancer trial in node-positive breast cancer comparing the addition of docetaxel to doxorubicin with doxorubicin-based chemotherapy. J Clin Oncol 2013;31:860-7.

6. Watanabe T, Hida AI, Inoue N, et al. Abundant tumor infiltrating lymphocytes after primary systemic chemotherapy predicts poor prognosis in estrogen receptor-positive/HER2-negative breast cancers. Breast Cancer Res Treat 2018;168:135-45.

7. Wu S, Zhou J, Ren Y, et al. Tumor location is a prognostic factor for survival of Chinese women with T1-2N0M0 breast cancer. Int J Surg 2014;12:394-8.

8. Niu S, Wen G, Ren Y, et al. Predictive Value of Primary Tumor Site for Loco-regional Recurrence in Early Breast Cancer Patients with One to Three Positive Axillary Lymphadenophy. J Cancer 2017;8:2394-400.

9. Yang J, Tang S, Zhou Y, et al. Prognostic implication of the primary tumor location in early-stage breast cancer: focus on lower inner zone. Breast Cancer 2018;25:100-7.

10. Lukesova L, Vrana D, Svach I, et al. Prognostic Influence of Internal Mammary Node Drainage in Patients with Early-stage Breast Cancer. Anticancer Res 2016;36:66416.

11. Courdi A, Chamorey E, Ferrero JM, et al. Influence of internal mammary node irradiation on long-term outcome and contralateral breast cancer incidence in node-negative breast cancer patients. Radiother Oncol 2013;108:259-65.

12. Lim ST, Choi JE, Kim SJ, et al. Prognostic implication of the tumor location according to molecular subtypes in axillary lymph node-positive invasive ductal cancer in a Korean population. Breast Cancer Res Treat 2016;156:473-83.

13. Ji F, Xiao WK, Yang CQ, et al. Tumor location of the central and nipple portion is associated with impaired survival for women with breast cancer. Cancer Manag Res 2019;11:2915-25.

14. Altundag K. Molecular Subtypes and Lower Inner Quadrant Tumors in Breast Cancer: Debate Is Still Ongoing. Clin Breast Cancer 2017;17:e165.

15. Zhu J, Jiao D, Guo X, et al. Predictive factors and prognostic value of pathologic complete response of ipsilateral supraclavicular lymph nodes in breast cancer after neoadjuvant chemotherapy. Ann Transl Med 2019;7:666.

16. Colleoni M, Zahrieh D, Gelber RD, et al. Site of primary tumor has a prognostic role in operable breast cancer:the 
international breast cancer study group experience. J Clin Oncol 2005;23:1390-400.

17. Yang K, Kim H, Choi DH, et al. Optimal radiotherapy for patients with internal mammary lymph node metastasis from breast cancer. Radiat Oncol 2020;15:16.

18. Aleknavi ius E, Atko ius V, Kuzmickien I, et al. Postmastectomy internal mammary nodal irradiation:a long-term outcome. Medicina (Kaunas) 2014;50:230-6.

19. Kim K, Jeong Y, Shin KH, et al. Impact of Regional Nodal Irradiation for Breast Cancer Patients With Supraclavicular and/or Internal Mammary Lymph Node Involvement: A Multicenter, Retrospective Study (KROG 16-14). Cancer Res Treat 2019;51:1500-8.

20. Thorsen LB, Thomsen MS, Berg M, et al. CTplannedinternal mammary node radiotherapy in the DBCG-IMN study: benefit versus potentially harmful effects. Acta Oncol 2014;53:1027-34.

21. Dess RT, Liss AL, Griffith KA, et al. Ischemic Cardiac Events Following Treatment of the Internal Mammary Nodal Region Using Contemporary Radiation Planning Techniques. Int J Radiat Oncol Biol Phys 2017;99:114653.

22. Piroth MD, Baumann R, Budach W, et al. Heart toxicity from breast cancer radiotherapy: Current findings, assessment, and prevention. Kardiale Toxizität durch Strahlentherapie bei Brustkrebs: Aktuelle Ergebnisse, Bewertung und Prävention. Strahlenther Onkol 2019;195:1-12.

23. Zhou W, Kuang T, Han X, et al. Prognostic role of lymphocyte-to-monocyte ratio in pancreatic neuroendocrine neoplasms. Endocr Connect 2020;9:28998.

24. Song L, Zhu J, Li Z, et al. The prognostic value of the lymphocyte-to-monocyte ratio for high-risk papillary thyroid carcinoma. Cancer Manag Res 2019;11:8451-62.

25. Huszno J, Kolosza Z. Prognostic value of the neutrophillymphocyte, platelet-lymphocyte and monocytelymphocyte ratio in breast cancer patients. Oncol Lett 2019;18:6275-83.

26. Ni XJ, Zhang XL, Ou-Yang QW, et al. An elevated peripheral blood lymphocyte-to-monocyte ratio predicts favorable response and prognosis in locally advanced breast cancer following neoadjuvant chemotherapy. PLoS One 2014;9:e111886.

27. Ji H, Xuan Q, Yan C, et al. The prognostic and predictive value of the lymphocyte to monocyte ratio in luminal-type breast cancer patients treated with CEF chemotherapy. Oncotarget 2016;7:34881-9.
28. Jia W, Wu J, Jia H, et al. The Peripheral Blood NeutrophilTo-Lymphocyte Ratio Is Superior to the Lymphocyte-ToMonocyte Ratio for Predicting the Long-Term Survival of Triple-Negative Breast Cancer Patients. PLoS One 2015;10:e0143061.

29. Zenan H, Zixiong L, Zhicheng Y, et al. Clinical prognostic evaluation of immunocytes in different molecular subtypes of breast cancer. J Cell Physiol 2019;234:20584-602.

30. Ji H, Xuan Q, Zhang Q, et al. The prognostic and predictive value of the lymphocyte to monocyte ratio in luminal-type breast cancer patients treated with CEF chemotherapy. Oncotarget 2016;7:34881-9.

31. Huszno J, Kołosza Z, Mrochem-Kwarciak J, et al. Prognostic Value of the Neutrophil-Lymphocyte, PlateletLymphocyte, and Monocyte-Lymphocyte Ratios in Male Breast Cancer Patients. Oncology 2020;98:487-92.

32. Bun A, Fujimoto Y, Higuchi T, et al. Prognostic Significance of Neutrophil-to-lymphocyte Ratio in Luminal Breast Cancers With Low Levels of Tumourinfiltrating Lymphocytes. Anticancer Res 2020;40:287180.

33. Yersal Ö, Çetinkünar S, Aktimur R, et al. Neutrophil/ Lymphocyte and Platelet/Lymphocyte Ratios are Not Different among Breast Cancer Subtypes. Asian Pac J Cancer Prev 2017;18:2227-31.

34. Cullinane C, Creavin B, O'Leary DP, et al. Can the Neutrophil to Lymphocyte Ratio Predict Complete Pathologic Response to Neoadjuvant Breast Cancer Treatment? A Systematic Review and Meta-analysis. Clin Breast Cancer 2020. [Epub ahead of print].

35. Rivas M, Acevedo F, Dominguez F, et al. The Neutrophil to Lymphocyte Ratio Predicts the Response to Neoadjuvant Chemotherapy in Luminal B Breast Cancer. Asian Pac J Cancer Prev 2019;20:2209-12.

36. Dieci MV, Criscitiello C, Goubar A, et al. Prognostic value of tumor-infiltrating lymphocytes on residual disease after primary chemotherapy for triple-negative breast cancer: a retrospective multicenter study. Ann Oncol 2014;25:611-8.

37. Hwang HW, Jung H, Hyeon J, et al. A nomogram to predict pathologic complete response (pCR) and the value of tumor-infiltrating lymphocytes (TILs) for prediction of response to neoadjuvant chemotherapy (NAC) in breast cancer patients. Breast Cancer Res Treat 2019;173:255-66.

38. Hamy AS, Bonsang-Kitzis H, De Croze D, et al. Interaction between Molecular Subtypes and Stromal Immune Infiltration before and after Treatment in Breast Cancer Patients Treated with Neoadjuvant Chemotherapy. Clin Cancer Res 2019;25:6731-41. 
39. Denkert C, von Minckwitz G, Darb-Esfahani S, et al. Tumour-infiltrating lymphocytes and prognosis in different subtypes of breast cancer: a pooled analysis of 3771 patients treated with neoadjuvant therapy. Lancet Oncol 2018;19: 40-50.

40. Tjan-Heijnen VC, Van Hellemond IE, Peer PG, et al. Abstract S1-03: First results from the multicenter phase III DATA study comparing 3 versus 6 years of anastrozole after 2-3 years of tamoxifen in postmenopausal women with hormone receptor-positive early breast cancer. Cancer
Res 2017. DOI: 10.1158/1538-7445.SABCS16-S1-03.

41. Wei J, Jiang Y, Shao Z, et al. The survival benefit of postmastectomy radiotherapy for breast cancer patients with T1-2N1 disease according to molecular subtype. Breast 2020;51:40-9.

42. Bräutigam E, Track C, Seewald DH, et al. Medial tumor localization in breast cancer--an unappreciated risk factor? Strahlenther Onkol 2009;185:663-8.

(English Language Editor: R. Scott)

Cite this article as: Song $\mathrm{X}, \mathrm{Ma} \mathrm{J}$, Zhang $\mathrm{H}$, Zhang Q. Prognostic significance of the primary tumor site and immune indexes in patients with estrogen receptor-positive, human epidermal growth factor receptor-2-negative breast cancer. Gland Surg 2020;9(5):1450-1468. doi: 10.21037/gs-20-622 